\title{
Constructing the secular architecture of the solar system
}

\section{The giant planets}

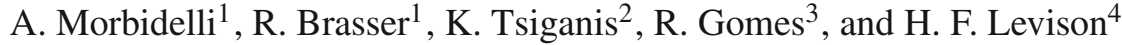 \\ 1 Dep. Cassiopee, University of Nice - Sophia Antipolis, CNRS, Observatoire de la Côte d'Azur, 06304 Nice, France \\ e-mail: brasser_astro@yahoo.com \\ 2 Department of Physics, Aristotle University of Thessaloniki, Thessaloniki, Greece \\ 3 Observatório Nacional, Rio de Janeiro, RJ, Brazil \\ 4 Southwest Research Institute, Boulder, CO, USA \\ Received 13 July 2009 / Accepted 31 August 2009
}

ABSTRACT

\begin{abstract}
Using numerical simulations, we show that smooth migration of the giant planets through a planetesimal disk leads to an orbital architecture that is inconsistent with the current one: the resulting eccentricities and inclinations of their orbits are too low. The crossing of mutual mean motion resonances by the planets would excite their orbital eccentricities but not their orbital inclinations. Moreover, the amplitudes of the eigenmodes characterising the current secular evolution of the eccentricities of Jupiter and Saturn would not be reproduced correctly, and only one eigenmode is excited by resonance-crossing. We show that, at the very least, encounters between Saturn and one of the ice giants (Uranus or Neptune) need to have occurred to reproduce the current secular properties of the giant planets, in particular the amplitude of the two strongest eigenmodes in the eccentricities of Jupiter and Saturn.
\end{abstract}

Key words. planets and satellites: formation - solar system: formation

\section{Introduction}

The formation and evolution of the solar system is a longstanding open problem. Of particular importance is the issue of the origin of the orbital eccentricities of the giant planets. Even though these are low compared to those of most extrasolar planets discovered so far, they are nevertheless high compared to what is expected from formation and evolution models.

Giant planets are expected to be born on quasi-circular orbits because low relative velocities with respect to the planetesimals in the disk are a necessary condition to allow the rapid formation of their cores (Kokubo \& Ida 1996, 1998; Goldreich et al. 2004). Once the giant planets have formed, their eccentricities evolve under the effects of their interactions with the disc of gas. These interactions can, in principle, enhance the eccentricities of very massive planets (Goldreich \& Sari 2003), but for moderate-mass planets they have a damping effect. In fact, numerical hydro-dynamical simulations (Kley \& Dirksen 2006; D'Angelo et al. 2006) show that only planets of masses higher than 2-3 Jupiter masses that are initially on circular orbits are able to excite an eccentricity in the disk and, in response, to become eccentric themselves. Planets of Jupiter-mass or less have their eccentricities damped. Accounting for turbulence should not change the result significantly: the eccentricity excitation due to turbulence is only in the order of 0.01 for a 10 Earth mass planet and decreases rapidly with increasing mass of said planet (Nelson 2005). By comparison, the mean eccentricities of Jupiter and Saturn are 0.045 and 0.05 respectively.

In addition, the interactions between Jupiter and Saturn, as they evolve and migrate in the disk of gas, should not lead to a significant enhancement of their eccentricities. Figure 1 shows a typical evolution of the Jupiter-Saturn pair, from Masset \& Snellgrove (2001). The top panel shows the evolution of the semi-major axes, where Saturn's semi-major axis is depicted by the upper curve and that of Jupiter is the lower trajectory. Initially far away, Saturn swiftly approaches Jupiter, possibly passing across their mutual 2:1 resonance (at approximately $9000 \mathrm{yr}$ in the figure), and is eventually trapped in the 3:2 resonance. At this point, the migration of both planets slows down slightly and then reverses. Morbidelli \& Crida (2007) argued that this dynamical evolution explains why Jupiter did not migrate all the way to the Sun in our System. Pierens \& Nelson (2008) convincingly demonstrated that the trapping in the 3:2 resonance is the only possible outcome for the Jupiter-Saturn pair. The lower panel of Fig. 1 shows the evolution of the eccentricities of both planets, where Saturn's eccentricity is depicted by crosses and that of Jupiter by bullets. Both eccentricities remain low all the time. The burst of the eccentricities associated with the passage through the 2:1 resonance at approximately $9000 \mathrm{yr}$ is rapidly damped. Once trapped in the 3:2 resonance, the equilibrium eccentricities are approximately 0.003 for Jupiter and 0.01 for Saturn, i.e. five to ten times smaller than their current values.

Once the gas has dispersed from the system, the giant planets are still expected to migrate, due to their interaction with a planetesimal disk (Fernandez \& Ip 1984; Malhotra 1993, 1995; Hahn \& Malhotra 1999; Gomes et al. 2004). While migration in the gas disk causes the planets to approach each other (Morbidelli et al. 2007), migration in the planetesimal disk causes the planets to diverge i.e. it increases the ratio between the orbital periods (Fernandez \& Ip 1984). In this process, the orbital eccentricities are damped by a mechanism known as "dynamical friction" (e.g. Stewart \& Wetherill 1988). Figure 2 provides an example of the eccentricity evolution of Jupiter (bullets) and Saturn (crosses) which are initially at 5.4 and 8.7 AU with their current eccentricities (initial conditions typical of Malhotra 1993, 1995; Hahn \& Malhotra 1999). They migrate, together with Uranus and Neptune, through a planetesimal disk carrying in total $50 M_{\oplus}$. The disk is simulated using 10000 tracers 

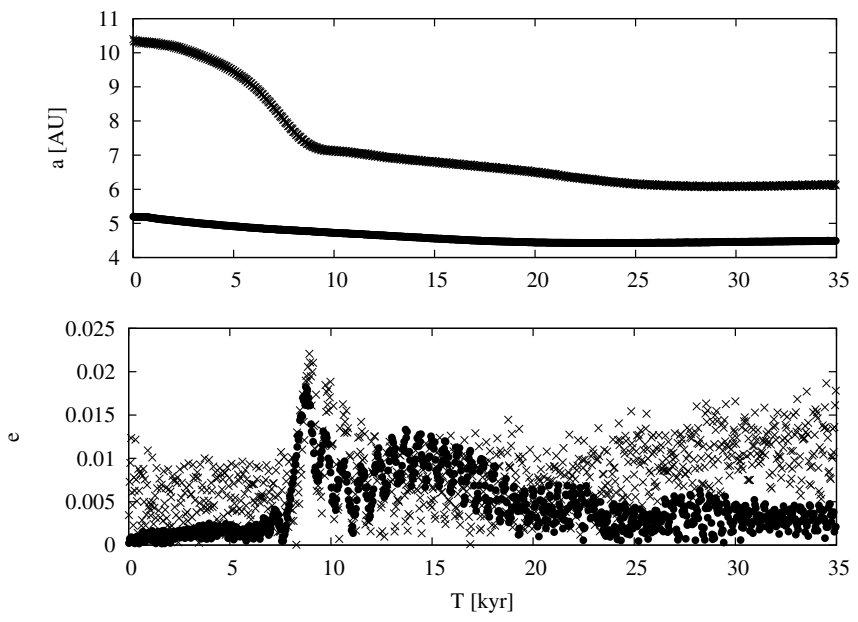

Fig. 1. The evolution of Jupiter (bullets) and Saturn (crosses) in the gas disk. Taken from Morbidelli \& Crida (2007), but reproducing the evolution shown in Masset \& Snellgrove (2001).

(see Gomes et al. 2004, for details). The figure shows the evolution of the eccentricities of Jupiter and Saturn: both are rapidly damped below 0.01 . Thus, a smooth radial migration through the planetesimal disk, as originally envisioned by Malhotra (1995) cannot explain the current eccentricities (nor the inclinations) of the orbits of the giant planets.

Then how did Jupiter and Saturn acquire their current eccentricities? In Tsiganis et al. (2005), the foundation paper for a comprehensive model of the evolution of the outer Solar System - often called the Nice model - it is argued that the current eccentricities were achieved when Jupiter and Saturn passed across their mutual 2:1 resonance, while migrating in divergent directions under the interactions with a planetesimal disk. They indeed showed that the mean eccentricities of Jupiter and Saturn are adequately reproduced during the resonance crossing (see electronic supplement of Tsiganis et al. 2005, or Fig. 5 below), as well as their orbital separations and mutual inclinations. However, the mean values of the eccentricities do not properly describe the secular dynamical architecture of a planetary system: the eccentricities of the planets oscillate with long periods, because of the mutual secular interactions among the planets. A system of $N$ planets has $N$ fundamental frequencies in the secular evolution of the eccentricities, and the amplitude of each mode - or, at least that of the dominant ones - should be reproduced in a successful model. We remind that Tsiganis et al. (2005) never checked if the Nice model reproduces the secular architecture of the giant planets (we will show below that it does) nor if this is achieved via the 2:1 resonance crossing (we will show here that it is not).

In this paper we make an abstraction of the Nice model, and investigate which events in the evolution of the giant planets are needed to achieve the current secular architecture of the giant planet system. We start in Sect. 2 by reviewing what this secular architecture is and how it evolves during migration, in the case where no mean motion resonances are crossed. In Sect. 3 we investigate the effect of the passage through the 2:1 resonance on the secular architecture of the Jupiter-Saturn pair. As we will see, this resonance crossing alone, although reproducing the mean eccentricities of both planets, does not reproduce the frequency decomposition of the secular system. In Sect. 4 we discuss the effect of multiple mean motion resonance crossings between Jupiter and Saturn, showing that this is still not

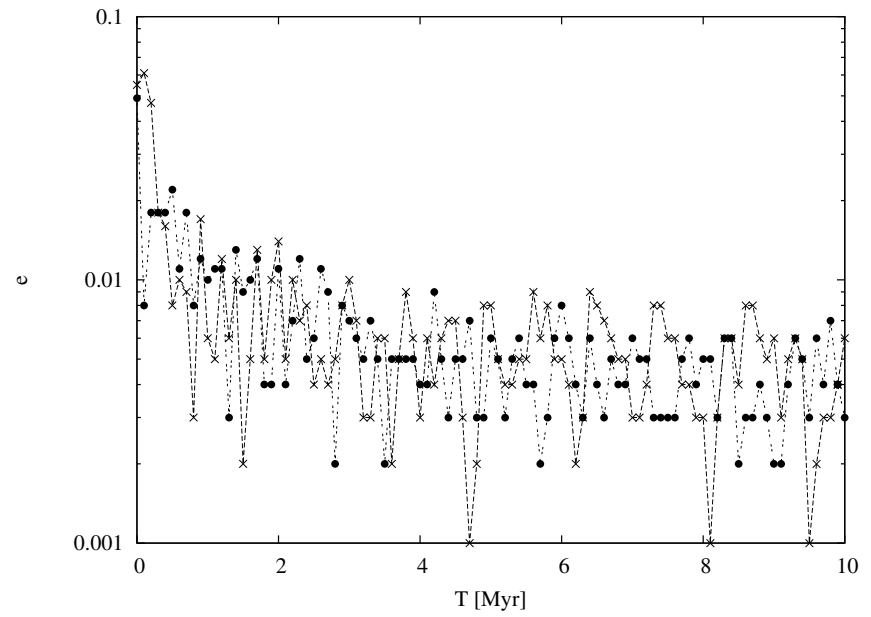

Fig. 2. The evolution of the eccentricities of Jupiter (bullet) and Saturn (crosses) as they migrate through a 50 Earth masses planetesimal disk. From Gomes et al. (2004).

enough to achieve the good secular solution. In Sect. 5 we examine the role of a third planet, with a mass comparable to that of Uranus or Neptune. We first consider the migration of this third planet on a circular orbit, then on an eccentric orbit and finally we discuss the consequences of encounters between this planet and Saturn. We show that encounters of Saturn with the ice giant lead to the correct secular evolution for the eccentricities of Jupiter and Saturn. In Sect. 6 we return to the Nice model, verify its ability to reproduce the current secular architecture of the planetary system and discuss other models that could, in principle, be equally successful in this respect. Although this paper is mostly focused on the Jupiter-Saturn pair and the evolution of their eccentricities, in Sect. 7 we briefly discuss the fate of Uranus and Neptune and the excitation of inclinations. The case of the terrestrial planets will be discussed in a second paper. The results are then summarised in Sect. 8.

\section{Secular eccentricity evolution of the Jupiter-Saturn pair}

One can study the secular dynamics of a pair of planets as described in Michtchenko \& Malhotra (2004). In that case the planets are assumed to evolve on the same plane and are far from mutual mean motion resonances. The Hamiltonian describing their interaction is averaged over the mean longitudes of the planets. This averaged Hamiltonian describes a two-degreesof-freedom system, whose angles are the longitudes of perihelia of the two planets: $\varpi_{1}$ and $\varpi_{2}$. The D'Alembert rules (see Chap. 1 of Morbidelli 2002), ensure that the Hamiltonian depends only on the combination $\Delta \varpi \equiv \varpi_{1}-\varpi_{2}$. Thus, the system is effectively reduced to one degree of freedom, which is integrable. This means that, in addition to the value of the averaged Hamiltonian itself, which will improperly be called "energy" hereafter, the system must have a second constant of motion. Simple algebra on canonical transformations of variables allows one to prove that this constant is

$$
K=m_{1}^{\prime} \sqrt{\mu_{1} a_{1}}\left(1-\sqrt{1-e_{1}^{2}}\right)+m_{2}^{\prime} \sqrt{\mu_{2} a_{2}}\left(1-\sqrt{1-e_{2}^{2}}\right)
$$

where $\mu_{i}=G\left(M+m_{i}\right), m_{i}^{\prime}=m_{i} M /\left(M+m_{i}\right), M$ is the mass of the star, $G$ is the gravitational constant and $m_{i}, a_{i}$ and $e_{i}$ are the mass, semi-major axis and eccentricity of each planet. Note 

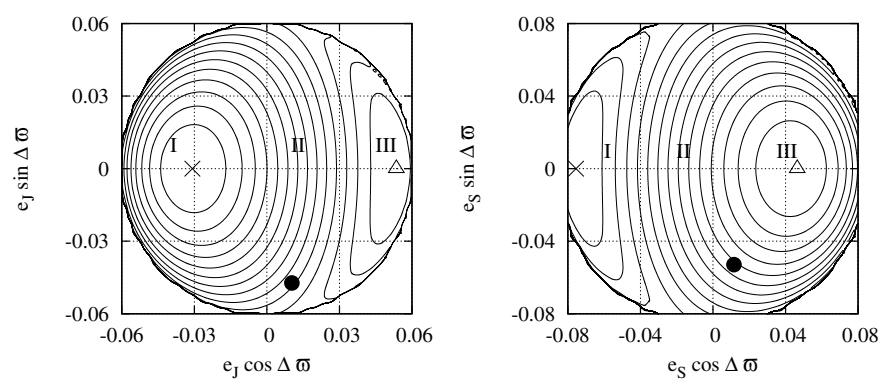

Fig. 3. Global illustration of the secular dynamics of the Jupiter-Saturn system. The bullets represent the current values of $e_{\mathrm{J}}, e_{\mathrm{S}}$ and $\Delta \varpi$.

that $K$, called angular momentum deficit, actually measures the deviation in angular momentum for an eccentric two-planet system, with respect to a system of two planets, both on circular orbits, with the same values of $a_{i}$. Now the global secular dynamics of the system can be illustrated by plotting level curves of the energy over manifolds defined by the condition that $K$ is constant.

Figure 3 shows the result for the Jupiter-Saturn system. The value of $K$ that we have chosen corresponds to the current masses, semi-major axes and eccentricities of these planets. The left panel illustrates the dynamics in the coordinates $e_{\mathrm{J}} \cos \Delta \varpi$ and $e_{\mathrm{J}} \sin \Delta \varpi$, while the right panel uses the coordinates $e_{\mathrm{S}} \cos \Delta \varpi$ and $e_{\mathrm{S}} \sin \Delta \varpi$, where $e_{\mathrm{J}}$ refers to the eccentricity of Jupiter and $e_{\mathrm{S}}$ to the eccentricity of Saturn. The bullets represent the current configuration of the Jupiter-Saturn system. We stress that the two panels are just two representations of the same dynamics. The same level curves of the energy are plotted in both panels. Thus, the $n$th level curve counting from the triangle in the left panel corresponds to the $n$th level curve counting from the triangle in the right panel. Indeed, the dot representing the current Jupiter-Saturn configuration is on the 5th level curve away from the triangle on each panel. The secular evolution of the system has to follow the energy level curve that passes through the dot. The other energy curves show the secular evolution that Jupiter and Saturn would have had, if the system were modified relative to the current configuration, preserving the current value of $K$. We warn the reader that the dynamics illustrated in this figure is not very accurate from a quantitative point of view because we have neglected the effects of the nearby 5:2 mean motion resonance between Jupiter and Saturn. Nevertheless all the qualitative aspects of the real dynamics are correctly reproduced.

We remark that the global secular dynamics of the JupiterSaturn system is characterised by the presence of two stable equilibrium points, one at $\Delta \varpi=0$ (marked by a triangle in Fig. 3) and one at $\Delta \varpi=\pi$ (marked by a cross). Thus, there are three kinds of energy level curves along which the Jupiter-Saturn system could evolve: those along which $\Delta \varpi$ librates around $\pi$ (type I), those along which $\Delta \varpi$ circulates (i.e. assumes all values from 0 to $2 \pi$; type II) and those along which $\Delta \varpi$ librates around 0 (type III). Notice that while type II curves wrap around the stable equilibrium at $\Delta \varpi=\pi$ in the left panel, the curves wrap around the stable equilibrium at $\Delta \varpi=0$ in the right panel. This means that during the circulation of $\Delta \varpi$, the eccentricity of Jupiter has a maximum when $\Delta \varpi=\pi$ while that of Saturn has a maximum when $\Delta \varpi=0$. The real Jupiter-Saturn system has this type of evolution.

We stress that there is no critical curve (separatrix) separating the evolutions of type I, II and III. By critical curve we mean a trajectory passing though (at least) one unstable equilibrium
Table 1. Frequencies and phases for the secular evolution of Jupiter and Saturn on their current orbits.

\begin{tabular}{ccc}
\hline \hline Frequency & Value $\left({ }^{\prime \prime} / \mathrm{yr}\right)$ & Phase $\left(^{\circ}\right)$ \\
\hline$g_{5}$ & 4.26 & 30.67 \\
$g_{6}$ & 28.22 & 128.11 \\
\hline
\end{tabular}

Table 2. Coefficients $M_{j, k}$ of the Lagrange-Laplace solution for the Jupiter-Saturn system. The coefficients of the terms with frequencies other than $g_{5}$ and $g_{6}$ are omitted.

\begin{tabular}{rcc}
\hline \hline$j\rangle^{k}$ & 5 & 6 \\
\hline 5 & 0.0442 & 0.0157 \\
6 & 0.0330 & 0.0482 \\
\hline
\end{tabular}

point, along which the travel time is infinite; an example is the curve separating the libration and circulation regimes in a pendulum. In this respect, speaking of "resonance" when $\Delta \varpi$ librates, as it is sometimes done when discussing the secular dynamics of extra-solar planets, is misleading because the word "resonance", in the classical dynamical systems and celestial mechanics terminology, implies the existence of such a critical curve.

In addition to using phase portraits, the secular dynamics of the Jupiter-Saturn system, or any pair of planets with small eccentricities, can also be described using the classical LagrangeLaplace theory (see Chap. 7 in Murray \& Dermott 1999). This theory, which is in fact the solution of the averaged problem described above, in the linear approximation, states that the eccentricities and longitudes of perihelia of the pair of planets evolve as:

$e_{\mathrm{J}} \cos \varpi_{\mathrm{J}}=M_{5,5} \cos \alpha_{5}-M_{5,6} \cos \alpha_{6}$

$e_{\mathrm{J}} \sin \varpi_{\mathrm{J}}=M_{5,5} \sin \alpha_{5}-M_{5,6} \sin \alpha_{6}$

$e_{\mathrm{S}} \cos \varpi_{\mathrm{S}}=M_{6,5} \cos \alpha_{5}+M_{6,6} \cos \alpha_{6}$

$e_{\mathrm{S}} \sin \varpi_{\mathrm{S}}=M_{6,5} \sin \alpha_{5}+M_{6,6} \sin \alpha_{6}$

where $\alpha_{5}=g_{5} t+\beta_{5}$ and $\alpha_{6}=g_{6} t+\beta_{6}$. Here $g_{5}$ and $g_{6}$ are the eigenfrequencies of the system, while $\beta_{5}$ and $\beta_{6}$ are their phases at $t=0$. In Eq. (2) all $M_{j, k}>0$. Tables 1 and 2 report the values of all the coefficients, obtained from the Fourier analysis of the complete 8-planet numerical solution (Nobili et al. 1989).

There is a one-to-one correspondence between the relative amplitudes of the coefficients $M_{j, k}$ and the three types of secular evolution illustrated in Fig. 3. We detail this relationship below, in order to achieve a better understanding of the planetary evolutions illustrated in the next sections.

From Eq. (2) the evolution of $e_{\mathrm{J}} \cos \Delta \varpi$ and $e_{\mathrm{S}} \cos \Delta \varpi$ (the quantities plotted on the $x$-axes of the panels in Fig. 3) are:

$$
\begin{aligned}
e_{\mathrm{J}} \cos \Delta \varpi= & {\left[M_{5,5} M_{6,5}-M_{5,6} M_{6,6}\right.} \\
& \left.+\left(M_{5,5} M_{6,6}-M_{5,6} M_{6,5}\right) \cos \left(\alpha_{5}-\alpha_{6}\right)\right] / e_{\mathrm{S}} \\
e_{\mathrm{S}} \cos \Delta \varpi= & {\left[M_{5,5} M_{6,5}-M_{5,6} M_{6,6}\right.} \\
& \left.+\left(M_{5,5} M_{6,6}-M_{5,6} M_{6,5}\right) \cos \left(\alpha_{5}-\alpha_{6}\right)\right] / e_{\mathrm{J}}
\end{aligned}
$$

where

$e_{\mathrm{J}}=\sqrt{M_{5,5}^{2}+M_{5,6}^{2}-2 M_{5,5} M_{5,6} \cos \left(\alpha_{5}-\alpha_{6}\right)}$

and

$e_{\mathrm{S}}=\sqrt{M_{6,5}^{2}+M_{6,6}^{2}+2 M_{6,5} M_{6,6} \cos \left(\alpha_{5}-\alpha_{6}\right)}$. 
When $\alpha_{5}-\alpha_{6}=0$ one has

$e_{\mathrm{J}} \cos \Delta \varpi=\left(M_{5,5}-M_{5,6}\right) \operatorname{sign}\left(M_{6,5}+M_{6,6}\right)$

$e_{\mathrm{S}} \cos \Delta \varpi=\left(M_{6,5}+M_{6,6}\right) \operatorname{sign}\left(M_{5,5}-M_{5,6}\right)$,

where $\operatorname{sign}()$ is equal to -1 if the argument of the function is negative, +1 if it is positive and 0 if it is zero. Instead, when $\alpha_{5}-\alpha_{6}=\pi$ one has

$e_{\mathrm{J}} \cos \Delta \varpi=\left(M_{5,5}+M_{5,6}\right) \operatorname{sign}\left(M_{6,5}-M_{6,6}\right)$

$e_{\mathrm{S}} \cos \Delta \varpi=\left(M_{6,5}-M_{6,6}\right) \operatorname{sign}\left(M_{5,5}+M_{5,6}\right)$.

Now, suppose that the amplitude corresponding to the $g_{5}$ frequency is zero, i.e. $M_{5,5}=M_{6,5}=0$. Then, the dependence of Eq. (3) on $\alpha_{5}-\alpha_{6}$ vanishes and, from Eq. (6) or Eq. (7), one sees that the system is located at the equilibrium point at $\Delta \varpi=\pi$, so that $e_{\mathrm{J}}=M_{5,6}$ and $e_{\mathrm{S}}=M_{6,6}$ (the point marked by a cross in Fig. 3).

Let us now gradually increase the amplitudes of the $g_{5}$ mode, relative to that of the $g_{6}$ one. This implies increasing $M_{5,5}$ and $M_{6,5}$ at the same rate, while keeping $M_{5,6}$ and $M_{6,6}$ fixed. Initially, when $M_{5,5}$ and $M_{6,5}$ are small compared to $M_{5,6}$ and $M_{6,6}$, all quantities in Eqs. (6) and (7) are negative, and therefore the evolution of the system follows an energy level curve of type $\mathrm{I}$, along which $\Delta \varpi$ librates around $\pi$. The distance of this curve from the equilibrium point, which we call amplitude of oscillation hereafter, is directly proportional to $M_{5,5}$ or $M_{6,5}$.

Since $M_{5,6}<M_{6,6}$ and $M_{6,5}<M_{5,5}$, then when $M_{5,5}=M_{5,6}$ one has $M_{6,5}<M_{6,6}$. This implies that increasing the amplitude of the $g_{5}$ mode eventually brings us to the situation where $M_{5,5}$ becomes larger than $M_{5,6}$, but $M_{6,5}$ is still less than $M_{6,6}$. Now the value of $e_{\mathrm{J}} \cos \Delta \varpi$ at $\alpha_{5}-\alpha_{6}=0$ i.e. $M_{5,5}-M_{5,6}$ becomes positive. When additionally $\alpha_{5}-\alpha_{6}=\pi$ its value remains negative, i.e. $-\left(M_{5,5}+M_{5,6}\right)$. Thus, the system now evolves on an energy curve of type II, along which $\Delta \varpi$ circulates. Notice that the value of $e_{\mathrm{S}} \cos \Delta \varpi$ at $\alpha_{5}-\alpha_{6}=\pi$ remains negative, while the value at $\alpha_{5}-\alpha_{6}=0$ jumps from $-\left(M_{6,5}+M_{6,6}\right)$ to $\left(M_{6,5}+M_{6,6}\right)$. Thus, the level curve in the right panel of Fig. 3 flips from one looping around the equilibrium point at $\Delta \varpi=\pi$ to one looping around the equilibrium at $\Delta \varpi=0$.

Further increasing the amplitude of the $g_{5}$ mode relative to that of the $g_{6}$ mode eventually results in $M_{6,5}$ also becoming larger than $M_{6,6}$. Now, all quantities in Eqs. (6) and (7) are positive, which means the system follows an energy level curve of type III, along which $\Delta \varpi$ librates around 0 . Notice that the value of $e_{\mathrm{J}} \cos \Delta \varpi$ at $\alpha_{5}-\alpha_{6}=\pi$ jumps from $-\left(M_{5,5}+M_{5,6}\right)$ to $\left(M_{5,5}+M_{5,6}\right)$, which means that the level curve in the left panel of Fig. 3 flips from one going around the equilibrium point at $\Delta \varpi=\pi$ to one going around the equilibrium at $\Delta \varpi=0$.

Finally, when the amplitude of the $g_{6}$ mode is zero, the system is on the stable equilibrium at $\Delta \varpi=0$ (the cross in Fig. 3).

Below we discuss how the secular dynamics of the planets changes as they migrate away from each other and are also submitted to dynamical friction, exerted by the planetesimal population.

\subsection{Migration and the evolution of the secular dynamics}

Let's imagine two planets migrating, without passing through any major mean motion resonance. A good example could be Jupiter and Saturn migrating from a configuration with orbital period ratio $P_{\mathrm{S}} / P_{\mathrm{J}}$ slightly higher than 2 to their current configuration, with $P_{\mathrm{S}} / P_{\mathrm{J}}$ slightly lower than 2.5 .

The migration causes the semi-major axis ratio between the planets to change. This affects the values of the coefficients $M_{j, k}$, since they depend explicitly on the above ratio;
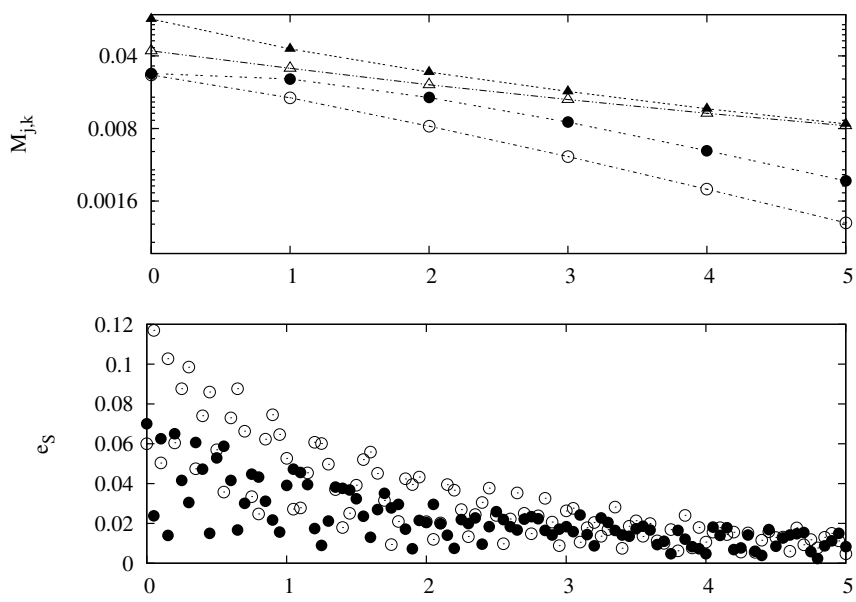

Fig. 4. Effect of eccentricity damping on the evolution of Jupiter and Saturn. In this experiment the damping force is applied to Saturn only. The top panel shows the coefficients $M_{5,5}$ (filled circles), $M_{5,6}$ (open circles), $M_{6,5}$ (filled triangles) and $M_{6,6}$ (open triangles) as a function of time. The bottom panel shows the eccentricities of Saturn (open circles) and Jupiter (filled circles).

in turn, this affects the global portrait of secular dynamics. However, if the migration is slow enough, the amplitude of oscillation around the equilibrium point is preserved as an adiabatic invariant (Neishtadt 1984; Henrard 1993). More precisely, it can be demonstrated that the conserved quantity is

$J=\oint m_{\mathrm{J}}^{\prime} \sqrt{G \mu_{\mathrm{J}} a_{\mathrm{J}}}\left(1-\sqrt{1-e_{\mathrm{J}}^{2}}\right) \mathrm{d} \Delta \varpi$,

which is the action conjugate to $\Delta \varpi$, and where $\oint$ denotes the integral over a closed energy curve (i.e. a bounded trajectory) that characterises the secular motion of the two planets (as in Fig. 3), if migration is frozen. Therefore, during migration, the planets would react to the slow changes in the global dynamical portrait by passing from one energy curve to another in such a way as to preserve the quantity in Eq. (8), i.e. the oscillation amplitude around the stable equilibrium point remains constant. Since for Jupiter and Saturn this amplitude is related to $M_{5,5}$, it turns out that any smooth migration should not have changed this coefficient significantly. In the next section the additional effect of dynamical friction is analysed.

\subsection{Dynamical friction and the evolution of the secular dynamics}

Dynamical friction is the mechanism by which gravitating objects of different masses exchange energy so as to evolve towards an equipartition of energy of relative motion (Saslaw 1985). For a system of planets embedded in a massive population of small bodies, the eccentricities and inclinations of the former are damped, while those of the latter are excited (Stewart \& Wetherill 1988).

In principle, each planet might suffer dynamical friction with different intensity, owing to a different location inside the planetesimal disk. However, the planets are connected to each other through their secular dynamics, so that dynamical friction, even if acting in an unbalanced way between the planets, turns out to have a systematic net effect.

To illustrate this point, consider again the Jupiter-Saturn system of Fig. 3 and suppose that dynamical friction is applied only 
to Saturn. The eccentricity of Saturn is damped, so the value of $K$ is reduced. Consequently, the location of the two stable equilibrium points has to move towards $e=0$. If the adiabatic invariance of Eq. (8) held, the amplitude of oscillation around the equilibrium point would be preserved, eventually turning a libration of $\Delta \varpi$ into a circulation. However, the adiabatic invariance does not hold in this case. The reason is that dynamical friction damps the eccentricity of Saturn, and therefore damps both the $M_{6,6}$ and the $M_{6,5}$ coefficients. Since the $M_{5,5}$ and $M_{6,5}$ coefficients are related, $M_{5,5}$ is also damped. In other words, the amplitude of oscillation around the equilibrium point is damped, and so the value of $J$ given in Eq. (8) decays with time.

As a check, we have run a simple numerical experiment. We have considered a Jupiter-Saturn system with semi-major axes 5.4 and 8.85 , with relatively eccentric orbits and large amplitude $\left(60^{\circ}\right)$ of apsidal libration around $\Delta \varpi=180^{\circ}$. We have integrated the orbits using the Wisdom-Holman (Wisdom \& Holman 1991) method, with the code Swift-WHM (Levison \& Duncan 1994). We used a time-step of $0.1 \mathrm{y}$ and modified the equations of motion so that a damping term is included for the eccentricity of Saturn only. Figure 4 shows the result. The bottom panel shows the evolutions of the eccentricities of the two planets, where $e_{\mathrm{S}}$ is represented by open circles and $e_{\mathrm{J}}$ by filled circles: both are damped and decay with time at the same rate. The top panel shows the amplitudes of the coefficients of Eq. (2), i.e. $M_{5,5}$ (filled circles), $M_{5,6}$ (open circles), $M_{6,5}$ (filled triangles) and $M_{6,6}$ (open triangles), computed at six different points in time. The computations were performed, by applying Fourier analysis to the time series produced in six short-time integrations, where no eccentricity damping was applied. As one can see, all coefficients decrease with time at comparable rates.

Thus, we conclude that, whatever the initial configuration of the planets, smooth migration and dynamical friction cannot increase the amplitude of the $g_{5}$ term and cannot turn the libration of $\Delta \varpi$ into a circulation. This result will be relevant in the next section.

\section{The effect of the passage through the 2:1 resonance}

We now consider the migration of Jupiter and Saturn, initially on quasi-circular orbits, through their mutual 2:1 mean motion resonance. Tsiganis et al. (2005) argued that this passage through the resonance is responsible for the acquisition of the current eccentricities of the two planets.

Figure 5 shows the effect of the passage through this resonance, starting from circular orbits. The simulation is again done using the Swift-WHM integrator, but in this case the equations of motion are modified so as to induce radial migration to the planets, with a rate decaying as $\exp (-t / \tau)$. No eccentricity damping is imposed. In practice, at every timestep $h$ the velocity of each planet is multiplied by a quantity $(1+\beta)$, with $\beta$ being proportional to $h \exp (-t / \tau)$. For Jupiter $\beta$ is negative and for Saturn it is positive, so that the two planets migrate inwards and outwards respectively, as observed in realistic $N$-body simulations (Fernandez \& Ip 1984; Hahn \& Malhotra 1999; Gomes et al. 2004). We chose $\tau=1$ Myr.

The top panel of Fig. 5 shows the ratio of the orbital periods of Saturn $\left(P_{\mathrm{S}}\right)$ and Jupiter $\left(P_{\mathrm{J}}\right)$ as a function of time. We stop the simulation well before $P_{\mathrm{S}} / P_{\mathrm{J}}$ achieves the current value, to emphasise the effect of the $2: 1$ resonance crossing. The middle panel shows $\Delta \varpi$ as a function of time. The bottom panel shows the evolutions of the eccentricities of Jupiter and Saturn,
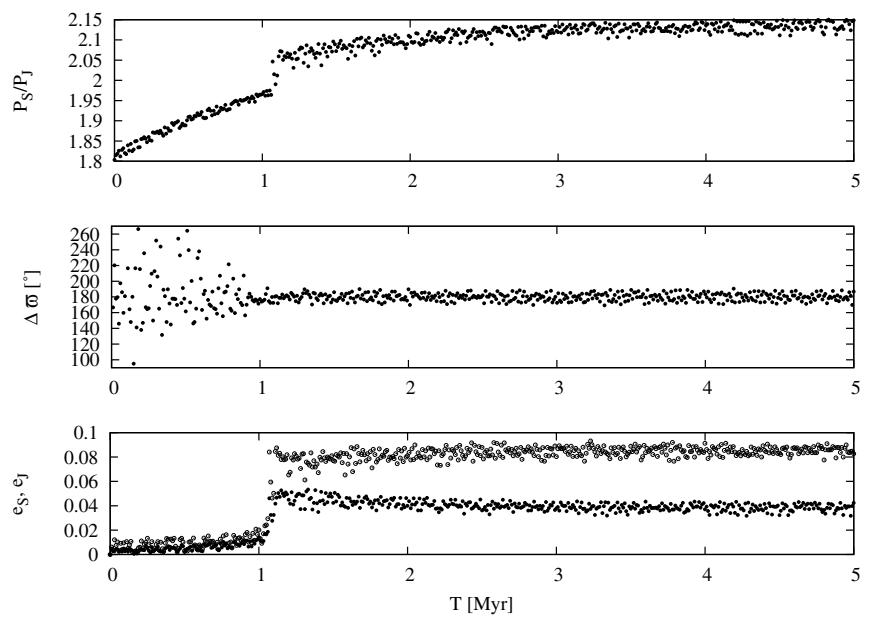

Fig. 5. The evolution of Jupiter and Saturn, as they pass across their mutual 2:1 resonance. In the bottom panel, Saturn's eccentricity is the upper curve and Jupiter's is the lower one.
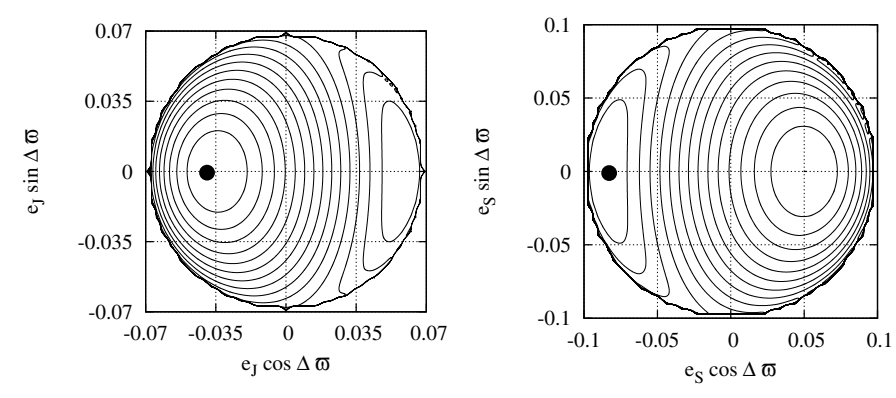

Fig. 6. Similar to Fig. 3 but after the 2:1 resonance crossing.

where the bottom trajectory corresponds to Jupiter and the top curve corresponds to Saturn. We notice that the orbital period ratio abruptly jumps across the value of 2 . Correspondingly, the eccentricities of Saturn and Jupiter jump to $\sim 0.07$ and $\sim 0.045$, which, as noticed by Tsiganis et al. (2005), are quite close to the current mean eccentricities of the two planets. During the subsequent migration, the eccentricity of Saturn increases somewhat and that of Jupiter decreases respectively. The two planets enter into apsidal anti-alignment (i.e. $\Delta \varpi$ librates around 180 degrees) shortly before the resonance passage and the libration amplitude shrinks down to $\sim 10^{\circ}$ as the eccentricities of the two planets grow. The crossing of the $2: 1$ does not seem to significantly affect the libration amplitude, which remains of the order of $\sim 10^{\circ}$ during the post-resonance-crossing migration (see also Ćuk 2007). As a result of this narrow libration amplitude, the eccentricities of Jupiter and Saturn do not show any sign of secular oscillation. In practice, Jupiter and Saturn are located at the stable equilibrium point of their secular dynamics, as shown in Fig. 6. This is very different from the current situation (compare with Fig. 3). Table 3 reports the values of the $M_{i, k}$ coefficients of Eq. (2) at the end of the simulation. The $M_{5,5}$ and $M_{6,5}$ coefficients, related to the amplitude of oscillation around the equilibrium point as explained in Sect. 2, are very small; they are more than an order of magnitude smaller than their current values. As discussed in Sect. 2.1, they would not increase during the subsequent migration of the planets because they behave as adiabatic invariants. Even worse, they would decrease if dynamical friction were applied. 
Table 3. Coefficients $M_{j, k}$ of the Jupiter-Saturn secular system at the end of the simulation illustrated in Fig. 5.

\begin{tabular}{rcc}
\hline \hline$j \backslash^{k}$ & 5 & 6 \\
\hline 5 & 0.00272 & 0.00275 \\
6 & 0.0378 & 0.0854 \\
\hline
\end{tabular}

Table 4. Values of $M_{5,5}$ in Jupiter after the 2:1 resonance crossing with Saturn as a function of the migration e-folding time, $\tau$.

\begin{tabular}{cc}
\hline \hline$\tau[\mathrm{Myr}]$ & $M_{5,5} \times 10^{-3}$ \\
\hline 1 & 3.55 \\
2 & 4.41 \\
5 & 7.49 \\
10 & 7.02 \\
20 & 5.05 \\
\hline
\end{tabular}

Simulations that we performed assuming longer values of $\tau$ (i.e. slower migration rates) lead to the same result. The eccentricities of Jupiter and Saturn after the 2:1 resonance crossing are approximately the same as in Fig. 5. The amplitude of libration of $\Delta \varpi$ is always between 6 and 15 degrees, with no apparent correlation on $\tau$. Table 4 recapitulates the results, for what concerns the values of the $M_{5,5}$ coefficient, which are always comparably small. Thus, we conclude that the 2:1 resonance crossing, although it explains the current mean values of the eccentricities of Jupiter and Saturn, cannot by itself explain the current secular dynamical structure of the system.

Let us now provide an interpretation of the behaviour observed in the simulation. The dynamics of two planets in the vicinity of a mean motion resonance can be studied following Michtchenko et al. (2008). For the 2:1 resonance, the fundamental angles of the problem are

$\sigma_{\mathrm{J}}=\lambda_{\mathrm{J}}-2 \lambda_{\mathrm{S}}+\varpi_{\mathrm{J}}$

$\Delta \varpi=\varpi_{\mathrm{J}}-\varpi_{\mathrm{S}}$.

The motion of the first angle is conjugated with the motion of the angular momentum deficit $K$ of the planets, defined in Eq. (1). The motion of the second angle is conjugated with the motion of the quantity $Q_{\mathrm{S}}=m_{\mathrm{S}}^{\prime} \sqrt{G \mu_{\mathrm{S}} a_{\mathrm{S}}}\left(1-\sqrt{1-e_{\mathrm{S}}^{2}}\right)$. If the system is far from the resonance, the motion of $\sigma_{\mathrm{J}}$ can be averaged out. Then $K$ becomes a constant of motion and the secular dynamics described in the previous section is recovered. In particular, if the planets migrate slowly enough so that the adiabatic invariance of $J$ - see Eq. (8) - holds, the amplitude of oscillation around the stable equilibrium point of the secular dynamics has to remain constant. However, as migration continues, the approach to the mean motion resonance forces the location of the equilibrium point to shift to higher eccentricity. Thus, by virtue of a geometric effect, the apparent amplitude of libration of $\Delta \varpi$ has to shrink as the eccentricities increase. This is visible in Fig. 5 in the phase before the resonance crossing.

As the planets are approaching the resonance, the angle $\sigma_{\mathrm{J}}$ can no longer be averaged out in a trivial way. However, an adiabatic invariant can still be introduced, as long as the timescale for the motion of $\sigma_{\mathrm{J}}$ is significantly shorter than that of $\Delta \varpi$ and that migration changes the system on even longer time scales. This invariant is

$\mathcal{K}=\oint K \mathrm{~d} \sigma_{\mathrm{J}}$,
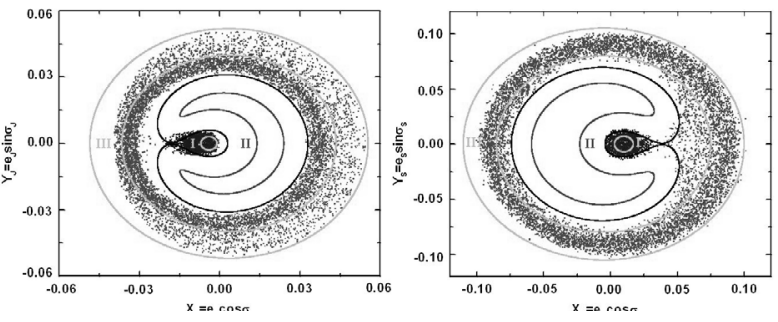

Fig. 7. Left: the evolution of $\sigma_{\mathrm{J}}$ and $e_{\mathrm{J}}$ in polar coordinates. Right: the evolution of $\sigma_{\mathrm{S}}$ and $e_{\mathrm{S}}$. Before the resonance is reached, both planets have nearly zero eccentricities (region I, black dots). When the resonance is reached, both planets jump to the corresponding Region III, along the $x$-axis and through the "X"-point of the critical curve (grey dots). From the supplementary material of Tsiganis et al. (2005).

where the integral is taken over a path describing the coupled evolution of $K$ and $\sigma_{\mathrm{J}}$, which is closed if $Q_{\mathrm{S}}$ and $\Delta \varpi$ are frozen (i.e. a trajectory of the so-called "frozen" system).

As shown in Michtchenko et al. (2008), for a pair of planets with the Jupiter-Saturn mass ratio and $e_{\mathrm{J}}<0.08$, the dynamics of the 2:1 resonance presents one critical curve, or separatrix, for the $K, \sigma_{\mathrm{J}}$ degree of freedom and no critical curve for the $Q_{\mathrm{s}}, \Delta \varpi$ degree of freedom. Thus, when the resonance is reached during the migration, the invariance of $\mathcal{K}$ is broken (Neishtadt 1984), since the two time-scales of the motion are no more well separated. As the planets are migrating in divergent directions, they cannot be trapped in the resonance (Henrard \& Lemaitre 1983). The resonant angle $\sigma_{\mathrm{J}}$ has to switch from clockwise to anti-clockwise circulation. Correspondingly, the quantity $\mathcal{K}$ has to jump to a higher value, that is the planets acquire an angular momentum deficit. How this new angular momentum deficit is partitioned between the two planets is difficult to compute a priori, because the dynamics are fully four-dimensional (i.e. two degrees of freedom) and, therefore, not integrable.

As shown in Fig. 7, there are clearly two regimes of motion in the portraits $Q_{\mathrm{J}}, \sigma_{\mathrm{J}}$ and $Q_{\mathrm{S}}, \sigma_{\mathrm{S}} \equiv \lambda_{\mathrm{J}}-2 \lambda_{\mathrm{S}}+\varpi_{\mathrm{S}}$ (remember that $e_{\mathrm{J}}^{2} \sim Q_{\mathrm{J}}$ and $e_{\mathrm{S}}^{2} \sim Q_{\mathrm{S}}$ ). Before the resonance crossing (black dots), the dynamics are confined in a narrow, off-centred region close to the origin of the polar coordinates. After the resonance crossing (grey dots), the dynamics fill a wide annulus, also asymmetric relative to the axis $\cos \sigma=0$. The curves in each panel are free hand illustrations of the dynamics near and inside a first-order mean motion resonance. The region filled by the black dots is bound by the inner loop of the critical curve (labelled I in the plot). The annulus filled by the grey dots is adhesive, at its inner edge, to the outer loop of the critical curve. The jump in eccentricity observed at the resonance crossing corresponds to the passage from the region inside the inner loop to that outside the outer loop.

Thus, in practice, it is as if each planet saw its own resonance: the one with critical angle $\sigma_{\mathrm{J}}$ for Jupiter and the one with critical angle $\sigma_{\mathrm{S}}$ for Saturn. The two resonances are just two slices of the same resonance, because only one critical curve exists (Michtchenko et al. 2008). This is the reason why the eccentricities of both planets jump simultaneously. The 2:1 resonance is structured by the presence of a periodic orbit, along which $\sigma_{\mathrm{J}}$ and $\Delta \varpi$ remain constant and are equal to 0 and $\pi$ respectively (Michtchenko et al. 2008). As $\Delta \varpi=\sigma_{\mathrm{J}}-\sigma_{\mathrm{S}}$, the phase portrait of the $\sigma_{\mathrm{J}}$ resonance and that of the $\sigma_{\mathrm{S}}$ resonance are rotated by 180 degrees with respect to each other. Thus, $Q_{\mathrm{J}}$ reaches a maximum when $\sigma_{\mathrm{J}}=0$ and $Q_{\mathrm{S}}$ has a maximum when $\sigma_{\mathrm{S}}=\pi$. Consequently, when the planets reach their maximal 
eccentricities and the $\sigma$ angles start to circulate anti-clockwise, $\Delta \varpi$ has to be $\sim 180^{\circ}$ (see Fig. 7). It is evident that the result of this transition through the resonance depends just on the resonance topology and not on the migration rate, as long as the latter is slow compared to the motion of the $\sigma$ angles (i.e. the adiabatic approximation holds, Neishtadt 1984).

After the resonance crossing, one can again average over $\sigma_{\mathrm{J}}$ and reduce the system to a one-degree of freedom secular system. As $\Delta \varpi=\pi$, Jupiter has to be on the negative $x$-axis of a diagram like that of the left panel of Fig. 6. Thus, the secular evolution of Jupiter will be an oscillation around the stable equilibrium at $\Delta \varpi=\pi$. The amplitude of this oscillation depends on the value of the eccentricity of Jupiter acquired at resonance crossing, relative to the value of the stable equilibrium of the secular problem. It turns out that, for the masses of Jupiter and Saturn, these two values are almost the same. Thus, the amplitude of oscillation is very small. We think that this is a coincidence and that, in principle, the situation does not have to be this way. In fact, we have verified numerically that the result depends on the individual masses of both planets, even for the same mass ratio. For instance, if the masses of Jupiter and Saturn are both reduced by a factor of 100 , the eccentricities of both planets jump to $\sim 0.01$ at resonance crossing, and this puts Jupiter on a secular trajectory that brings $e_{\mathrm{J}}$ down to 0 ; that is, the secular motion is now at the boundary between type-I and type-II, as defined in Sect. 2. This is caused by a different scaling of the jumps in $e_{\mathrm{J}}$ and $e_{\mathrm{S}}$ with respect to the planetary masses and to a different global secular dynamics in the vicinity of the resonance, which in turn is caused by a different relative importance of the quadratic terms in the masses.

Once the planets are placed relative to the portrait of their secular dynamics, their destiny is fixed. As they move away from the mean motion resonance, the secular portrait can change, in particular because the near-resonant perturbation terms that are quadratic in the masses rapidly decrease in amplitude. Hence the location of the equilibrium points can change, but the planets have to follow them adiabatically. This explains the slow monotonic growth of the eccentricity of Saturn and the decay of that of Jupiter, observed in the top panel of Fig. 5, while the amplitude of libration of $\Delta \varpi$ does not change (middle panel).

\section{Passage through multiple resonances}

Since the passage of Jupiter and Saturn through the 2:1 resonance, starting from initially circular orbits, produces a secular system that is incompatible with the current one, we now explore the effects of the passage of these planets through a series of resonances. This is done to determine whether or not such evolution could increase the value of $M_{j, 5}$ in both planets.

We place Jupiter and Saturn initially on quasi-circular orbits just outside their mutual 3:2 resonance. The choice of these initial conditions is motivated by the result that during the gas-disk phase, Saturn should have been trapped in the 3:2 resonance with Jupiter (Morbidelli et al. 2007; Pierens \& Nelson 2008). Once the gas disappeared from the system, the two planets should have been extracted from the resonance at low eccentricity, by the interaction with the planetesimals, and subsequently start to migrate.

In the above setting, our planets are forced to migrate through the 5:3, 7:4, 2:1, 9:4 and 7:3 resonances, ending up close to their current location in semi-major axis (i.e. slightly interior to the 5:2 resonance). In the migration equations we have chosen $\tau$ such that it takes about $40 \mathrm{Myr}$ to reach $P_{\mathrm{S}} / P_{\mathrm{J}} \approx 2.5$
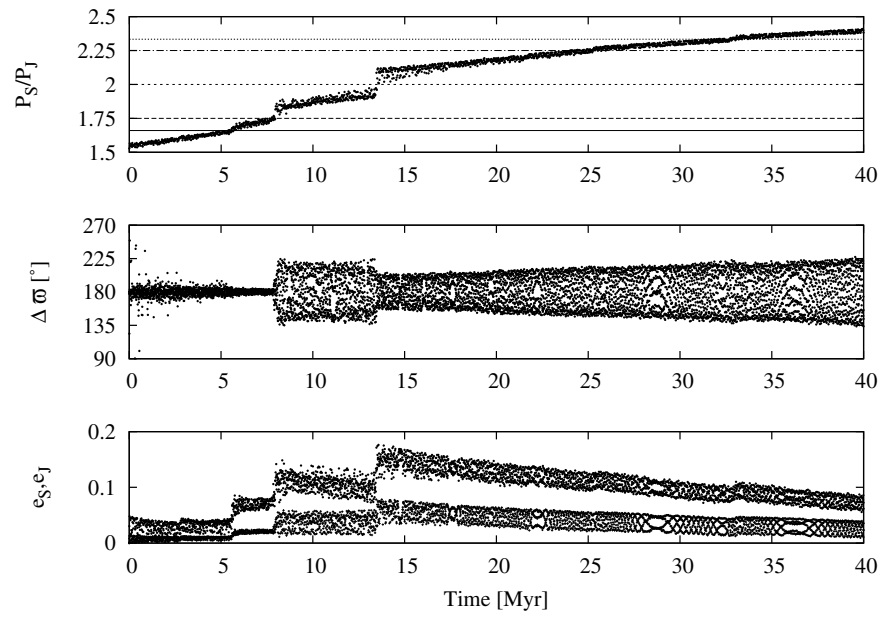

Fig. 8. Like Fig. 5, but for Jupiter and Saturn evolving through a sequence of mean motion resonances, from just outside their mutual 3:2 commensurability up to their current location.

although, as we saw before, the migration timescale has little influence on the resonant effects. The result of this experiment is shown in Fig. 8, which is similar in format to Fig. 5. The horizontal lines in the top panel denote the positions of the resonances mentioned above. Notice a distinct jump in the eccentricities of both planets at each resonance crossing. In order to prevent the system from becoming unstable we applied eccentricity damping to Saturn, so as to mimic the effect of dynamical friction and so that the planets reach final eccentricities that are similar to the current mean values of the two planets. The parameters for the simulation depicted in Fig. 8 are $\tau=25 \mathrm{Myr}$ and $\dot{e}_{\mathrm{S}}=-2 \times 10^{8} \mathrm{yr}^{-1}$. The effect of damping is visible in the eccentricity evolution, after the $2: 1$ resonance crossing; we will discuss the effect on the motion of $\Delta \varpi$ below.

The middle panel of Fig. 8 shows that the passage through the 7:4 resonance significantly increases the libration amplitude of $\Delta \varpi$. In fact, the amplitude of the $M_{5,5}$ term in (2), increases from $9 \times 10^{-4}$ before the resonance crossing, to 0.019 after the crossing. However, the passage through the $2: 1$ resonance shrinks the amplitude of libration of $\Delta \varpi$. This happens because the $2: 1$ resonance crossing, as we have seen in the previous section, does not enhance $M_{5,5}$ (it remains equal to 0.019 in this simulation) but does enhance the overall eccentricities of the planets. As explained earlier, this causes the amplitude of libration of $\Delta \varpi$ to decrease.

Notice from Fig. 8 that, after the 2:1 resonance crossing, the amplitude of libration of $\Delta \varpi$ starts to increase, slowly and monotonically. This is caused not by an enhancement of the amplitude of oscillation around the equilibrium point of the secular dynamics, but by the damping of the eccentricities of the planets. It is the opposite of what was just described before: a geometrical effect. In reality, the value of $M_{5,5}$ is decreased to 0.015 (from 0.019) during this evolution. Hence, at the end of the simulation, the amplitude of the $g_{5}$ mode is about a factor of 3 smaller than in the real secular dynamics of Jupiter and Saturn. Without eccentricity damping, the amplitude of the $g_{5}$ mode would have remained equal to $\sim 0.019$, still much smaller than in the current Jupiter-Saturn secular dynamics.

Several other experiments, where we slightly changed the initial conditions or the migration speed $\tau$, lead essentially to the same result. Thus, we conclude that the migration of Jupiter and Saturn through a sequence of mean motion resonances is not 
enough to achieve their current secular configuration. A richer dynamics is required, likely involving interactions with a third planet.

\section{Three-planet dynamics}

From the discussions and the examples reported above, it is quite clear that, to enhance the amplitudes of the $g_{5}$ mode, it is necessary that the eccentricity of Saturn receives a kick that is not counterbalanced by a corresponding increase in the eccentricity of Jupiter (or vice versa). This would indeed move the planets away from the stable equilibrium point of their secular dynamics, thus enhancing the amplitude of oscillation around this point and, consequently, $M_{j, 5}$. Given that Jupiter and Saturn are not alone in the outer solar system, in this section we investigate the effect that interactions with a third planet with a mass comparable to that of Uranus and Neptune, which we simply refer to as "Uranus", has on the Jupiter-Saturn pair. We first address the effects of the migration of Uranus on a quasi-circular orbit. Then we study the effects of its migration on an initially eccentric orbit and, finally, we address the problem of encounters among the planets.

\subsection{Migration of Uranus on a quasi-circular orbit}

The main mean motion resonance with Saturn that Uranus can go through is the $2: 1$. Thus, this is the resonance crossing that we focus on here. Given that, as we have seen in the previous sections, the effect of a passage through a mean motion resonance is quite insensitive to the migration rate, the initial location of the planets etc., the main issue that may potentially lead to different results is whether the crossing of the 2:1 Saturn-Uranus resonance happened before or after the putative crossing of the 2:1 Jupiter-Saturn resonance. Below we investigate each of these two cases.

We have performed a numerical experiment where we have the crossing of the Saturn-Uranus resonance happen first, with Saturn and Jupiter having initially a small orbital period ratio $\left(P_{\mathrm{S}} / P_{\mathrm{J}}=1.53\right)$, and Uranus and Saturn having an orbital period ratio $P_{\mathrm{U}} / P_{\mathrm{S}} \sim 1.95$. The exact initial locations are not important, as long as they do not change the order of the resonance crossings. All planets initially have circular orbits. The three planets are forced to migrate to their current positions with $\tau=5$ Myr. Eccentricity damping is imposed on Saturn and Uranus, to mimic dynamical friction, with forces tuned such that, at the end of the simulation, Uranus approximately reaches its current eccentricity.

Figure 9 shows the result. The top panel shows the pericentre $q$ and apocentre $Q$ of the planets which, from top to bottom, are Uranus, Saturn and Jupiter respectively. The separation among these curves gives a visual measure of the eccentricity of the orbit of the respective planet. The middle panel shows $\Delta \varpi$ for Jupiter and Saturn.

In this simulation, Uranus crosses the 2:1 resonance with Saturn at $t \sim 0.9$ Myr. This gives a kick to the eccentricity of Uranus (its $q, Q$-curves abruptly separate from each other) and, to a lesser extent, to the eccentricity of Saturn. This sudden increase in the eccentricity of Saturn moves the stable equilibrium point of the Jupiter-Saturn secular dynamics away from $e_{\mathrm{J}} \sim e_{\mathrm{S}} \sim 0$. However, the eccentricity of Jupiter does not receive an equivalent kick by this resonance crossing, so it remains close to zero. Consequently, Jupiter must start evolving secularly along a trajectory close to the boundary between type-I and typeII curves (see Sect. 2); in other words $M_{5,5} \sim M_{5,6}$. This is the
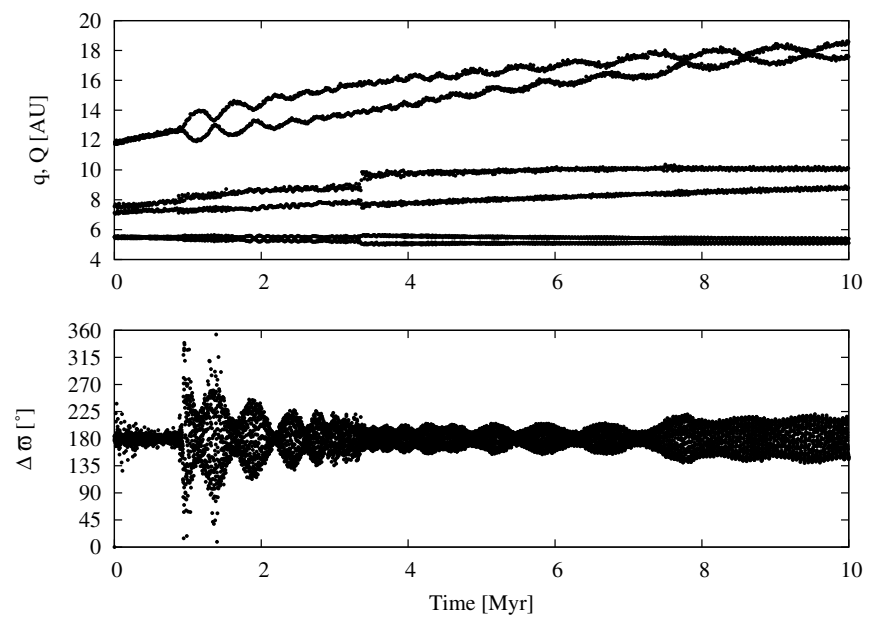

Fig. 9. A three-planet migration simulation, in which both the $2: 1$ resonance between Uranus and Saturn and the 2:1 resonance between Saturn and Jupiter are crossed.

reason why the amplitude of libration of $\Delta \varpi$ changes abruptly at the Uranus-Saturn resonance crossing, and reaches an amplitude of $\sim 180^{\circ}$.

The interim between 0.9 and $3.3 \mathrm{Myr}$ is characterised by large, long-periodic, oscillations of the eccentricity of Uranus, which correlate with the modulation of the amplitude of libration of $\Delta \varpi$. These oscillations have a frequency equal to $g_{5}-g_{7}$, where $g_{7}$ is the new fundamental frequency that characterises the extension of (2) to a three-planet system. Soon after the Uranus-Saturn resonance crossing, $g_{5}-g_{7}$ is slow compared to the frequencies themselves and therefore the oscillations have large amplitude. As Uranus departs from the resonance with Saturn, $g_{7}$ decreases; at the same time, $g_{5}$ increases, since Jupiter approaches the 2:1 resonance with Saturn. Hence, the oscillations with frequency $g_{5}-g_{7}$ becomes faster and its amplitude deceases. This sequence of increasingly shorter oscillations reduces the overall amplitude of libration of $\Delta \varpi$ to approximately 40 degrees.

At $t \sim 3.3$ Myr, Jupiter and Saturn cross their mutual 2:1 mean motion resonance, which has the effects that we discussed before. The amplitude of $M_{5,5}$ is roughly preserved in this resonance crossing, as already illustrated in Sect. 4. Its value at $t=4 \mathrm{Myr}$ is 0.010 , much larger than in Sect. 3 but still about a factor of four smaller than the current value. The $M_{5,5}$ coefficient receives an additional small enhancement at $t \sim 7.5 \mathrm{Myr}$, when Jupiter and Saturn cross their 7:3 resonance, but this does not change the substance of the result.

To reverse the order of the resonance crossings, we have run a second experiment, in which we placed Jupiter and Saturn just beyond their $2: 1$ resonance $\left(P_{\mathrm{S}} / P_{\mathrm{J}}=2.06\right)$, on orbits typical of those achieved by the $2: 1$ resonance crossing (see Sect. 3). This means that Jupiter and Saturn are in apsidal libration around $180^{\circ}$, with an amplitude of the $g_{5}$ mode that is small in both planets compared to the current value (see Table 3 ). Uranus was placed on a circular orbit at $a_{\mathrm{U}}=12.5$. Again the planets were forced to migrate to their current locations, with $\tau=5$ Myr. The 2:1 resonance crossing between Uranus and Saturn again kicked the eccentricity of Saturn, which in turn enhanced the amplitude of the $g_{5}$ term. In this case, the final value of the $M_{5,5}$ coefficient was 0.014 , i.e. similar to the previous experiment. 

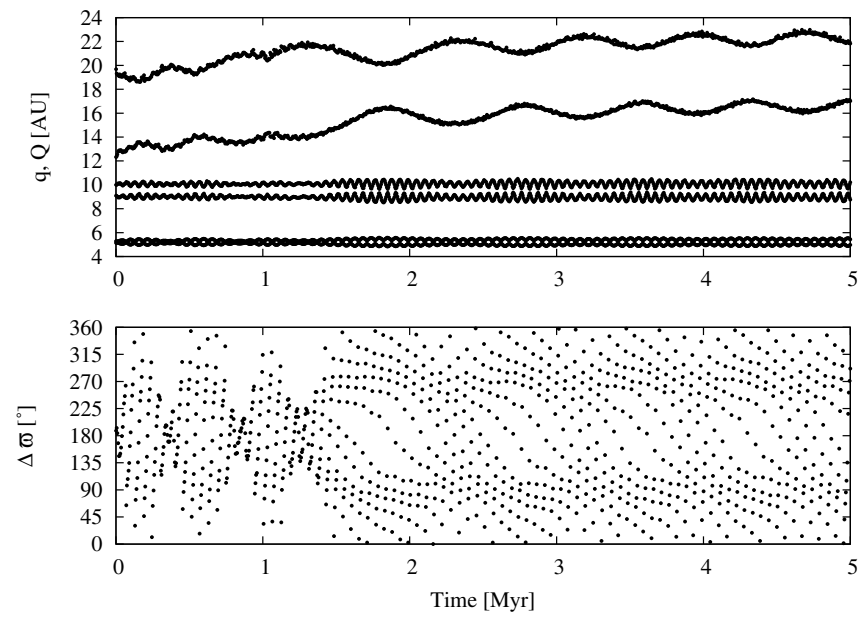

Fig. 10. A three-planet simulation, with Uranus initially on an eccentric orbit and migrating to its current location. No migration is imposed on Jupiter and Saturn.

Given the above results, we conclude that, no matter when the Uranus-Saturn 2:1 resonance crossing occurs, there is an enhancement of the amplitude of the $g_{5}$ term, as expected, but it is too small (by a factor of $\sim 3$ ) to explain the current Jupiter-Saturn secular system. It appears that the mass of Uranus is too small to provide enough eccentricity excitation on Saturn, when passing through a mean motion resonance with it.

\subsection{Migration of Uranus on an eccentric orbit}

In the current solar system, the proper frequency of perihelion of Uranus, $g_{7}$, is lower than $g_{5}$ (3.1 and $4.3^{\prime \prime} / \mathrm{yr}$, respectively). If Uranus was much closer to Saturn, however, $g_{7}$ had to be much higher too. For instance, if Uranus were just outside the 2:1 resonance with Saturn (say $a_{\mathrm{U}}=14.8 \mathrm{AU}$ and $a_{\mathrm{S}}=8.6 \mathrm{AU}$ ), $g_{7}$ was $\sim 6.5^{\prime \prime} / \mathrm{yr}$. One might then wonder if, during the migration of Uranus, the $g_{7}=g_{5}$ secular resonance could have occurred. On the other hand, if Jupiter was closer to Saturn, $g_{5}$ would have been higher as well; $g_{5}>6.5^{\prime \prime} / \mathrm{yr}$ if $P_{\mathrm{S}} / P_{\mathrm{J}} \lesssim 2.2$. Thus, the occurrence of the $g_{5}=g_{7}$ resonance depends on the locations of both Jupiter and Uranus, relative to Saturn.

To see the effect of this secular resonance, we performed an idealised experiment, in which we placed Jupiter at $a_{\mathrm{J}}=5.2 \mathrm{AU}$, Saturn at $a_{\mathrm{S}}=9.5 \mathrm{AU}$ and Uranus at $a_{\mathrm{U}}=16 \mathrm{AU}$ with an eccentricity of 0.25 . The initial eccentricities and $\Delta \varpi$ for Jupiter and Saturn were taken from a run, in which these planets passed through their mutual 2:1 resonance and migrated up to the locations reported above. Hence $\Delta \varpi$ would librate with very small amplitude, in the absence of Uranus. In this experiment the latter was forced to migrate towards its current location, with $\tau=2$ Myr. No migration was imposed on Jupiter and Saturn. Since initially $g_{5}=4.4^{\prime \prime} / \mathrm{yr}$ and $g_{7}>g_{5}$, the $g_{5}=g_{7}$ resonance crossing had to occur during this simulation. No eccentricity damping was applied to any of the planets in this run.

The result is shown in Fig. 10. In the first part of the simulation $(t<1.3 \mathrm{Myr})$ the amplitude of libration of $\Delta \varpi$, which is initially very small, suffers a large modulation, correlated with the oscillations of the eccentricity of Uranus. The dynamics here are analogous to what we described before, for the interim between the two mean motion resonance crossings in Fig. 9. At $t \sim 1.3 \mathrm{Myr}$, the $g_{5}=g_{7}$ resonance is crossed. This leads to an exchange of angular momentum between Uranus and Jupiter.
The eccentricity of Uranus decreases a bit, while the value of the $M_{5,5}$ coefficient is enhanced. As a response, $\Delta \varpi$ starts to circulate. The final value of $M_{5,5}$ is 0.04 , essentially matching the current value.

Although Jupiter has to be far from Saturn to have a genuine secular resonance crossing, we have found that more realistic simulations, in which Jupiter and Saturn are initially much closer to each other - so to be able to migrate in the correct proportion with respect to Uranus - can lead to interesting results as well. The reason is that, although from the beginning $g_{7}<g_{5}$, the two frequencies can become quasi-resonant; such interactions also allow for a significant transfer of eccentricity from Uranus to Jupiter and can excite the value of $M_{5,5}$ up to the current figure.

However, the reader should be aware that, while the effect of mean motion resonances is quite insensitive to parameters and initial conditions, in the case of a secular resonance, the outcome depends critically on a variety of issues. More precisely, the effects of the $g_{7}=g_{5}$ resonance, or quasi-resonance, must depend on the eccentricity of Uranus, the migration timescale $\tau$ and the position of $\varpi_{\mathrm{U}}$ relative to $\varpi_{\mathrm{J}}$, immediately before this resonant interaction. The reason for the first dependence is that $e_{\mathrm{U}}$ sets the strength of the secular resonance. The dependence on $\tau$ and $\varpi_{U}$ has to do with the fact that the timescale associated with a secular resonance is very long, of the order of 1 Myr. Thus, migration through a secular resonance, unlike migration through a mean motion resonance, is not an adiabatic process, at least for values of $\tau$ up to $10 \mathrm{Myr}$ that we are focusing on here. Thus, the time spent in the vicinity of the resonance and the values of the phases at which the planets enter the resonance have important impact on the resulting dynamics.

Given the above, we conclude that the secular interaction with an eccentric Uranus is a mechanism that is potentially capable of exciting the $g_{5}$ mode in the Jupiter-Saturn system to the observed level, but this mechanism is quite un-generic. Moreover, if we invoked an eccentric Uranus to explain the origin of the Jupiter-Saturn dynamical architecture, we would still need to explain how Uranus got so eccentric in first place. Finally, the $g_{7}=g_{5}$ secular resonance cannot alone explain the excitation of the planetary inclinations, which will be discussed in Sect. 7. For all these reasons, we continue our search for a better mechanism and consider below the effect of planetary encounters.

\subsection{Planetary encounters with Uranus}

Close encounters between Uranus and Saturn could potentially be a very effective mechanism for kicking the eccentricity of Saturn and enhancing the amplitude of the $g_{5}$ mode.

To investigate this, we have run a series of twenty simulations, where the initial semi-major axes of Jupiter and Saturn were chosen such that these two planets are just outside their 2:1 mean motion resonance $\left(P_{\mathrm{S}} / P_{\mathrm{J}}=2.06\right)$, on orbits typical of those achieved during the $2: 1$ resonance crossing (in apsidal antialignment with negligible oscillation amplitude). Uranus was placed on an orbit with semi-major axis ranging from $11.8 \mathrm{AU}$ to $13.4 \mathrm{AU}$ at $0.2 \mathrm{AU}$ intervals, with an initial eccentricity of 0.1 . This value of the eccentricity is similar to that achieved by Uranus under the secular forcing induced by Jupiter and Saturn. The system was then allowed to evolve under the mutual gravitational forces and external migration forces. For all simulations, the e-folding time for the migration forces was set at $5 \mathrm{Myr}$. Eccentricity damping was applied to Uranus and Saturn, the values being $\dot{e}_{\mathrm{S}}=-2 \times 10^{-8} / \mathrm{yr}$ and $\dot{e}_{\mathrm{U}}=-1.2 \times 10^{-7} / \mathrm{yr}$. The damping coefficient for Uranus was assumed to be six times larger 

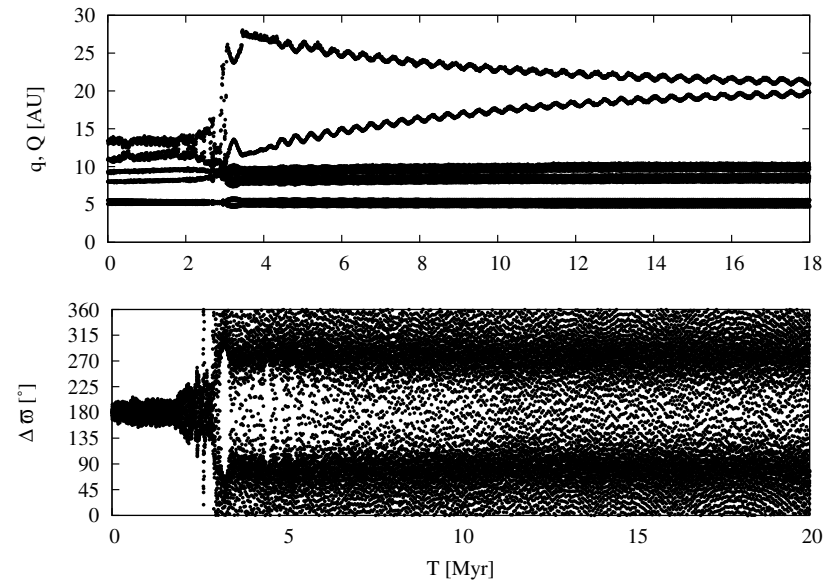

Fig. 11. Example of an encounter between Uranus and Saturn. The plot is similar to Fig. 9.

than that of Saturn because, in principle, Uranus is more affected by the planetesimal disk than the gas-giants. The strength of the damping term was calibrated so that the post-encounter evolution of the eccentricity of Uranus follows the one seen in the full $N$-body simulations of Tsiganis et al. (2005). These details are not very important because we focus here on the final secular dynamics of Jupiter and not on the final orbit of Uranus. The latter is very sensitive to the prescription of damping, but not the former, as we have seen in Sect. 2.2. Uranus was typically found to be scattered by Saturn (and sometimes by Jupiter). The simulations were stopped once the phase of encounters among the planets ended, either because Uranus was decoupled from the giant planets, due to eccentricity damping, or because it was ejected from the system.

The simulations that yielded the best results in terms of final $M_{5,5}$ value are those with initial semi-major axes of Uranus $a_{\mathrm{U}}<13$ AU. In these successful simulations, a total of four or $20 \%$, the average final value of $M_{5,5}$ was approximately 0.04 , in very good agreement with the current configuration. Figure 11 gives an example of evolution from one of these successful runs, and is similar to Figs. 9 and 10. As seen in our results, initially placing Uranus further away from Saturn results in encounters that are too weak to pump up the $g_{5}$ mode in Jupiter.

In summary, we conclude that encounters between Saturn and Uranus constitute an effective and quite generic mechanism for achieving a final secular evolution of the Jupiter-Saturn system that is consistent with their current state. Compared to all other mechanisms investigated in this paper, which either do not work or work only for an ad-hoc set of conditions, planet-planet scattering is our favoured solution to the problem of the origin of the secular architecture of the giant planet system. In Sect. 7 we provide further arguments in favour of this conclusion.

\section{The Nice model and its alternatives}

The work presented above shows that a combination of the effects provided by the $2: 1$ resonance crossing between Jupiter and Saturn and by encounters and/or secular interactions with an eccentric Uranus, can produce a Jupiter-Saturn system that behaves secularly like the real planets.

The 2:1 resonance crossing, the encounters among the planets and the high-eccentricity phases of Uranus and Neptune are essential ingredients of the Nice model (Tsiganis et al. 2005; Gomes et al. 2005; Morbidelli et al. 2007). Thus, we expect that

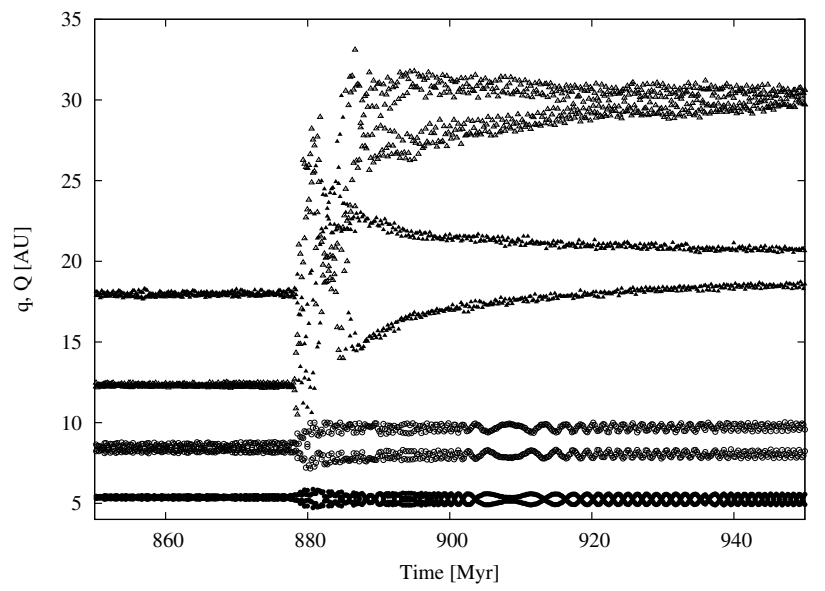

Fig. 12. Sample evolution of the giant planets in the Nice model (from Gomes et al. 2005). Each planet is represented by a pair of curves, showing the time evolution of their perihelion and aphelion distance.

this model not only reproduces the mean orbital eccentricities of the planets, as shown in Tsiganis et al. (2005), but also the correct architecture of secular modes. Curiously, this has never been properly checked before, and we do so in the following.

In Fig. 12, the pericentre and apocentre distance of the four giant planets are plotted as a function of time, in a simulation taken from Gomes et al. (2005) that adequately reproduces the current positions of the giant planets. The curve starting around 5 AU represents Jupiter, the one around 9 AU is Saturn, the trajectory at 12 AU is Uranus (who ends up switching positions with Neptune) and the uppermost curve at $16 \mathrm{AU}$ is Neptune (who ends up closer to the Sun than Uranus). This plot is a magnification around the time when Jupiter and Saturn cross their $2: 1$ resonance and the system becomes unstable. The plot shows the phase until all encounters had stopped, which in this case happened when the period ratio between Jupiter and Saturn was $P_{\mathrm{S}} / P_{\mathrm{J}}=2.23$. The final semi-major axes of the four planets are $\left(a_{\mathrm{J}}, a_{\mathrm{S}}, a_{\mathrm{U}}, a_{\mathrm{N}}\right)=(5.23,8.94,19.88,31.00)$, so that the largest "error" is in Saturn's orbit. A Fourier spectrum of Jupiter's eccentricity at the end of the simulation gives $M_{5,5}=0.027$, and $M_{5,6}=0.036$. The amplitude of the $g_{5}$ term is a bit small, but well within a factor of 2 from the real value. Another Nice-model run from the same Gomes et al. (2005) study gave an essentially identical result. However, a third simulation gave $M_{5,5}=0.059$, which is larger than the real value. In a fourth simulation, in which not only Saturn but also Jupiter encountered an ice giant, a value close to the current one was again recoverd, namely $M_{5,5}=0.037$. Given the chaotic nature of planetary encounters and that the resulting $M_{5,5}$ values are close to the current one (0.044) or even larger (e.g. 0.059), we conclude that the Nice model is able to reproduce the secular architecture of the JupiterSaturn system.

In principle, depending on the initial separations between Saturn and the innermost ice giant, the Nice model can also give planetary evolutions in which encounters between Saturn and an ice giant do not occur (only the ice giants encountering each other; see Tsiganis et al. 2005). These evolutions have been rejected already in Tsiganis et al. (2005), because they lead to final mean eccentricities (and inclinations) that are too low and an orbital separation between Saturn and Uranus that is too narrow at the end. They also lead to a value of $M_{5,5}$ that is much too small compared to the current value because the $2: 1$ resonance 
crossing alone is not capable of pumping the excitation of the $g_{5}$ mode, as we have seen earlier.

At this point, one might wonder whether the Nice model, in the version with Saturn-Uranus encounters, is the only model capable of this result. From the study reported in this paper, it seems likely that encounters among planets might be sufficient to excite the modes of the final secular system, without any need for Jupiter and Saturn crossing their mutual 2:1 resonance. In other words, one might envision a model where Jupiter and Saturn formed on circular orbits, well separated from each other in the beginning, so that $P_{\mathrm{S}} / P_{\mathrm{J}}$ was always larger than 2 . These planets then had close encounters with other planetary embryos, which at the end left them on eccentric orbits with both the $g_{5}$ and $g_{6}$ modes excited.

A single encounter of an embryo with one planet would not work because, by kicking the eccentricity of one planet and not of the other, it would produce a secular system with $M_{5,5} \sim M_{5,6}$ (if the embryo encountered Saturn) or with $M_{6,6} \sim M_{6,5}$ (if the embryo encountered Jupiter). The real system is different from these two extremes. However, multiple encounters with one planet or with both of them should do the job. To achieve an estimate of the mass of the planetary embryo that could excite the secular modes of Jupiter and Saturn up to the observed values, we have run four sets of four simulations each. In each run we considered Jupiter, Saturn and one embryo, initially on circular orbits. The mass of the embryo was 1, 5, 10 and 15 Earth masses respectively, for the four sets of simulations. The initial location of the embryo was $a_{\mathrm{e}}=7.2 \mathrm{AU}, 8.0 \mathrm{AU}, 10.1 \mathrm{AU}, 10.7 \mathrm{AU}$, for the four simulations in each set, whereas Jupiter and Saturn were initially at $a_{\mathrm{J}}=5.4 \mathrm{AU}$ and $a_{\mathrm{S}}=8.9 \mathrm{AU}$ in all cases. In most simulations, the embryo was eventually ejected from the Solar System: in two runs the embryo collided with Jupiter. The values of the $M_{5,5}$ and $M_{6,6}$ coefficients for each set of simulations are reported in Table 5. It turns out that the putative embryo had to be massive, of the order of $>10 M_{\oplus}$. We stress that multiple embryos with the same total mass would not do an equal job because the geometries of the encounters would be randomised leading to dynamical friction instead of excitation. In fact, we did the same experiment with 100 Mars mass objects instead of a unique 10 Earth-mass object; it resulted in $M_{5,5}$ and $M_{6,6}$ being smaller than 0.001, demonstrating that an ensemble of small objects could not have excited the relevant modes to their current states. In conclusion, for the excitation of the $M_{5,5}$ mode to reach its current value, Jupiter and Saturn should have encountered Uranus or Neptune or a putative third ice giant of comparable mass. Therefore, for what concerns the excitation of the secular Fourier modes of the planetary orbits, a generic scenario of global instability and mutual scattering of the four giant planets, as originally proposed by Thommes et al. (1999) would work; the passage of Jupiter and Saturn through their mutual 2:1 resonance, which is specific to the Nice model relative to Thommes et al. (1999) (or Thommes et al. 2007, in which Jupiter and Saturn are initially locked in the 2:1 resonance) is not necessary.

However, Pierens \& Nelson (2008) showed that the only possible final configuration achieved by Jupiter and Saturn in the gas disk is in their mutual 3:2 resonance. Unless alternative evolutions have been missed in that work, this result invalidates the initial planetary configurations considered in Thommes et al. (1999, 2007) and supports the Nice model, in particular in its newest version described in Morbidelli et al. (2007), where the four giant planets start locked in a quadruple resonance with $P_{\mathrm{S}} / P_{\mathrm{J}}=3: 2$.
Table 5. Values of $M_{5,5}$ in Jupiter and $M_{6,6}$ in Saturn after ejecting planetary embryos of various masses from various original locations.

\begin{tabular}{cccc}
\hline \hline$m_{\mathrm{e}}\left[M_{\oplus}\right]$ & $a_{\mathrm{e}}[\mathrm{AU}]$ & $M_{5,5} \times 10^{-3}$ & $M_{6,6} \times 10^{-3}$ \\
\hline 1 & 7.2 & 4.41 & 5.92 \\
1 & 8 & 1.34 & 3.17 \\
1 & 10.1 & 0.475 & 8.91 \\
1 & 10.7 & 8.97 & 8.56 \\
5 & 7.2 & 5.23 & 85.3 \\
5 & 8.0 & 15.0 & 57.3 \\
5 & 10.1 & 7.78 & 12.0 \\
5 & 10.7 & 7.49 & 20.2 \\
10 & 7.2 & 38.0 & 88.5 \\
10 & 8.0 & 22.5 & 15.1 \\
10 & 10.1 & 11.0 & 32.8 \\
10 & 10.7 & 66.0 & 28.7 \\
15 & 7.2 & 32.3 & 41.0 \\
15 & 8.0 & 20.3 & 28.9 \\
15 & 10.1 & 20.4 & 80.9 \\
15 & 10.7 & 14.8 & 152.1 \\
\hline
\end{tabular}

\section{Ice giants and inclination constraints}

Up to now we have focused our discussion on the secular evolution of the eccentricities of Jupiter and Saturn. However, the outer Solar System has two additional planets: Uranus and Neptune, which introduce the additional frequencies $g_{7}$ and $g_{8}$ in the secular evolution of the eccentricities. Thus, one should also be concerned about the correct excitation of the $g_{7}$ and $g_{8}$ modes in all planets. In addition, the planets have a rich secular dynamics in inclination, associated with the regression of their nodes. The excitation of the correct modes in the inclinations is a problem as crucial as that of the eccentricities.

The reason that we did not discuss these issues so far is because considerations based solely on the secular evolution of the eccentricities of Jupiter and Saturn have proved enough to guide us towards a solution, which is also valid in the more general problem. That is, these planetary encounters that are necessary to explain the Jupiter-Saturn secular architecture, can also explain the excitation of the $g_{7}$ and $g_{8}$ modes i.e. $M_{j, 7}$ and $M_{j, 8}$, and of the inclinations of the planets.

The excitation of the $g_{7}$ and $g_{8}$ modes does not appear to be very constraining. During the Saturn-Uranus and UranusNeptune encounters, the eccentricities of the ice giants become typically much larger than the current values. Thus, the combination of encounters and dynamical friction can produce a wide range of amplitudes of the $g_{7}$ and $g_{8}$ modes, including the current amplitudes. But we cannot exclude that other mechanisms, such as a sequence of mutual resonance crossing, could have led to the correct amplitudes as well.

Conversely, the inclination excitation is particularly interesting because it provides a strong, additional argument in favour of a violent evolution of the planets that involves mutual close encounters. In fact, the planets should form on essentially coplanar orbits, for the same reasons for which they should form on circular orbits: low relative velocities with respect to the planetesimals in the disk are necessary for the rapid formation of their cores. Once the planets are formed, tidal interactions with the gas disk damp the residual inclinations of the planets (Lubow \& Ogilvie 2001). After the disappearance of the gas, dynamical friction exerted by the remnant planetesimal disk would also damp the planetary inclinations. Thus, similar to the eccentricities, a relatively-late excitation mechanism is required to explain 
the inclinations of the planets. However, unlike the eccentricities, the passage across mean motion resonances does not significantly excite the inclinations because the resonant terms depending on the longitude of the nodes are at least quadratic in the inclinations. Secular resonances are also ineffective, if all planetary inclinations are initially low. The only mechanism by which inclinations can be efficiently increased is by close encounters. In fact, in the Nice model, the inclinations of Saturn, Uranus and Neptune, relative to the orbit of Jupiter, are well reproduced. A similar result holds also for the Thommes et al. (1999) model.

It is interesting to note that the eccentricities of the giant planets are about twice as large as the inclinations (respectively $\sim 0.05$ and $\sim 0.025$ radians or $\sim 1.5^{\circ}$ ). This is what one would expect, if both the eccentricities and the inclinations had been acquired by a combination of gravitational scattering and dynamical friction. Conversely, if encounters among the planets had never happened and the eccentricities had been acquired through specific resonance crossings, we would expect the planetary eccentricities to be much higher than the inclinations.

\section{Conclusion}

In this work we have demonstrated that the secular architecture of the giant planets, which have non-negligible eccentricities and inclinations and specific amplitudes of the modes in the eccentricity evolution of Jupiter and Saturn, could not have been achieved if the planets migrated smoothly through a planetesimal disk, as originally envisioned in Malhotra (1993; see also Malhotra 1995; Hahn \& Malhotra 1999; Gomes et al. 2004). Thus, we believe that the community should no longer consider the smooth migration model as a valid template for the evolution of the solar system. Even repeated passages through mutual resonances, that would have happened if the planetary system was originally more compact than envisioned in Malhotra's model, could not account for the orbital architecture of the giant planets, as we observe them today.

Instead, the outer planetary system had to evolve in a more violent way, in which the gas giants encountered the ice giants, or other rogue planets of equivalent mass. In this respect, the correct templates for the planetary evolution are the Nice model or the Thommes et al. model (or variants of these two). As we noted above, the Nice model seems to be, so far, more coherent and consistent in all its facets. We remark that encounters among the planets have been shown to also be an effective mechanism for the capture of the systems of irregular satellites (Nesvorny et al. 2007). These arguments, which are different and independent of those reported in this paper, also support a violent evolution scenario of the outer solar system.

In a forthcoming paper we will investigate the orbital dynamics of the terrestrial planets in the context of the evolution of the giant planets that we have outlined in this work.

Acknowledgements. This work is part of the Helmholtz Alliance's "Planetary evolution and life", which RB and AM thank for financial support. Exchanges between Nice and Thessaloniki have been funded by a PICS programme of France's CNRS, and R.B. thanks the host K.T. for his hospitality during a recent visit. RG thanks Brazil's CNPq and FAPERJ for financial support. H.F.L. thanks NASA's OSS and OPR programmes for support. Most of the simulations for this work were performed on the CRIMSON Beowulf cluster at OCA.

\section{References}

Ćuk, M. 2007, DPS, 39, 60

D’Angelo, G., Lubow, S., \& Bate, M. 2006 ApJ 652, 1698

Fernandez, J. A., \& Ip, W.-H. 1984, Icarus, 58, 109

Goldreich, P., \& Sari, R. 2003, ApJ, 585, 1024

Goldreich, P., Lithwick, Y., \& Sari, R. 2004, ApJ, 614, 497

Gomes, R., Morbidelli, A., \& Levison, H. 2004, Icarus, 170, 492

Gomes, R., Levison, H., Tsiganis, K., \& Morbidelli, A. 2005, Nature, 435, 466

Hahn, J., \& Malhotra, R. 1999, AJ, 117, 3041

Henrard, J., \& Lemaître, A. 1983, Icarus, 55, 482

Henrard, J. 1993, in Dynamics Reported New series, 2, 117

Kley, W., \& Dirksen, G. 2006, A\&A, 447, 369

Kokubo, E., \& Ida, S. 1996, Icarus, 123, 180

Kokubo, E., \& Ida, S. 1998, Icarus, 131, 171

Levison, H., \& Duncan, M. 1994, Icarus, 108, 18

Lubow, S., \& Ogilvie, G. 2001, ApJ, 560, 997

Malhotra, R. 1993, Nature, 365, 819

Malhotra, R. 1995, AJ, 110, 420

Masset, F., \& Snellgrove, M. 2001, MNRAS, 320, 55

Michtchenko, T., \& Malhotra, R. 2004, Icarus, 168, 237

Michtchenko, T., Beaugé, C., \& Ferraz-Mello, S. 2008, MNRAS, 391, 215

Morbidelli, A. 2002, Modern Celestial Mechanics - Aspects of Solar System Dynamics (Taylor \& Francis, UK).

Morbidelli, A., \& Crida, A. 2007, Icarus, 191, 158

Morbidelli, A., Tsiganis, K., Crida, A., Levison, H., \& Gomes, R. 2007, AJ, 134, 1790

Murray, C., \& Dermott, S. 1999, Solar System Dynamics (Cambridge, UK: Cambridge University Press)

Neishtadt, A. 1984, J. App. Math. Mech., 48, 133

Nelson, R. 2005, A\&A, 4431067

Nesvorný, D., Vokrouhlický, D., \& Morbidelli, A. 2007, AJ, 133, 1962

Nobili, A., Milani, A., \& Carpino, M. 1989, A\&A, 210, 313

Pierens, A., \& Nelson, R. 2008, A\&A, 482, 333

Saslaw, W. 1985, ApJ, 297, 49

Stewart, G., \& Wetherill, G. 1988, Icarus, 74, 542

Tsiganis, K., Gomes, R., Morbidelli, A., \& Levison, H. 2005, Nature, 435, 459

Thommes, E., Duncan, M., \& Levison, H. 1999, Nature, 402, 635

Thommes, E., Nilsson, L., \& Murray, N. 2007, ApJ, 656, 25

Wisdom, J., \& Holman, M. 1991, AJ, 102, 1528 